\title{
ESTUdo DAS CÉlulas DE DEFESA ATIVADAS NA DINÂMICA DO HIV
}

\author{
Camila Vianna de Magalhães*, Roberto Carlos Antunes Thomé*, Dayse Haime Pastore*, \\ HYUN MO YANG ${ }^{\dagger}$ \\ *CEFET/RJ - Centro Federal de Educação Tecnológica Celso Suckow da Fonseca \\ Av. Maracanã, 229 \\ Rio de Janeiro \\ 20271-110, RJ \\ Brasil \\ †UNICAMP - IMECC, Departamento de Matemática Aplicada \\ Caixa Postal 6065 \\ Campinas \\ CEP: $13081-970, S P$ \\ Brasil
}

Emails: milamagal@yahoo.com.br, rthome@cefet-rj.br, dpastore@cefet-rj.br, hyunyang@ime.unicamp.br

\begin{abstract}
We study a mathematical model that describes the dynamics of the spread of HIV in the human body. This model is introduced through a system of differential equations involving ordinary susceptible cells, infected cells, HIV, immune cells and cells of active defenses. The difference of these Model to others found in the literature are exactly two moments of activity of the immune system in the defense against HIV: cells without activation for HIV and the activated cells for HIV.
\end{abstract}

Keywords - HIV, Mathematical Model, Immune System

Resumo - Estudamos um modelo matemático que descreve a dinâmica de propagação do HIV no organismo humano. Este modelo é apresentado por meio de um sistema de equações diferenciais ordinárias que envolvem células suscetíveis, células infectadas, HIV, células de defesa e células de defesas ativas. A diferença desses modelo para outros encontrados na literatura são exatamente dois momentos de atuação do sistema imunológico na defesa contra o HIV: as células sem ativação para o HIV e as células ativadas para o HIV.

Palavras-chave- HIV, Modelagem Matemática, Sistema Imunológico.

\section{Introdução}

O vírus da Imunodeficiência Humana, também conhecido como $H I V$, (sigla em inglês para human immunodeficiency virus), é da família dos retrovírus e o responsável pela $A I D S$. A infecção pelo HIV resulta em uma doença crônica e progressiva, que pode levar à destruição do sistema imunológico. A evolução da doença se caracteriza por uma elevada taxa de replicação viral, que resulta na emergência de variantes virais mais virulentas. A infecção pelo HIV é, atualmente, delineada pela contagem do número de células $C D_{4}+$ pela quantidade de partículas virais no sangue (carga viral) e pelos sintomas clínicos. Nem todas as pessoas apresentam todos os estágios da doença, e o tempo entre a infecção e a manifestação dos diferentes quadros clínicos pode variar muito, dependendo do indivíduo.

Para se reproduzir, o HIV une-se à membrana de uma célula vital para o sistema imunológico, a T4. O vírus libera seu $R N A$ e uma enzima, a transcriptase reversa, com a qual fabrica o $D N A$ viral. O DNA viral entra no núcleo e une-se ao DNA da célula, assumindo o comando. O resultado dessa união é o DNA Pró-Viral que fabrica o $R N A$ mensageiro com o código genético do vírus.
O RNA mensageiro desloca-se para o citoplasma e produz os Vírions. Os Vírions saem da célula hospedeira como novos HIV's. Um único vírus gera muitos outros pontos para infectarem outras células (Amendoeira, 2009).

O modelo proposto apresenta-se com a introdução de uma nova variável que é chamada de células específicas de defesa ativadas. Isto deve-se explica-se, depois de alguns estudos e pesquisas, que é extremamente importante para o modelo uma vez que em nosso organismo já possuimos células de defesa estando contaminado ou não. Faz parte do conjunto de células de qualquer indivíduo, possuir células suscetíveis e células de defesa que estão prontas para nos defender desde uma simples infecção até algo mais grave. Com a contaminação $H I V$, o que ocorre é justamente a destruição dessas células que ficaram impedidas de nos defender de uma simples gripe podendo tornar-se algo muito mais periogoso ao nosso organismo. Sendo assim, uma vez contaminada, a pessoa passa a ter também, células infectadas, vírus e células especificas de defesa ativadas que estarão ali presentes com o intuito de tentar destruir exatamente o $H I V$. 


\section{Apresentação do modelo}

Quando o HIV invade o corpo humano, o alvo são as células de defesa T CD4+ presentes no organismo. Essas células, consideradas como "mensageiras", sinalizam a presença de um invasor para as outras células de defesa (B e T CD8+). As células T CD8+ são as que respondem a esse sinal para destruírem as células infectadas. A partir dessa resposta elas se tornam específicas para o HIV.

Neste trabalho, estamos propondo um novo modelo matemático para estudar a dinâmica do HIV no sistema imunológico humano. Estamos propondo modificações de vários modelos existentes na literatura (Perelson and Nelson, 1999; Komarova and Nowak, 2001; Nowak and May, 2000; Grégio et al., 2009). Uma delas é introdução de uma nova variável para melhor descrever a defesa do sistema imunológico.

Esse modelo é dado pelo sistema de equações diferenciais ordinárias que reproduziremos a seguir:

$$
\left\{\begin{aligned}
\dot{x} & =\lambda_{x}-\mu_{x} x-\beta_{v} x v \\
\dot{y} & =\beta_{v} x v-\mu_{y} y-p_{y} y z_{a} \\
\dot{v} & =k_{v} y-\mu_{v} v-p_{v} v z_{a} \\
\dot{z} & =\lambda_{z}-\mu_{z} z-\beta_{z} z v \\
\dot{z}_{a} & =\beta_{z} z v-\mu_{z} z_{a}
\end{aligned}\right.
$$

No sistema (1), a variável $z$ é a população de todas as células efetoras da resposta imunológica em repouso e a variável $z_{a}$ é a população dessas células ativadas no combate as células infectadas, que está respondendo com anticorpos.

O valores usados nas simulações, foram retirados dos artigos (Nowak and May, 2000; McLean, 2013; Perelson and Nelson, 1999)

Tabela 1: Condição Inicial

\begin{tabular}{lcl}
\hline Variáveis de Estado & Símbolo & Valor \\
\hline $\begin{array}{l}\text { Células T CD4+ existen- } \\
\text { tes no organismo (susce- }\end{array}$ & $x$ & $10^{3} \mathrm{~mm}^{-3}$ \\
tíveis) & & \\
$\begin{array}{l}\text { Células T CD4+ infecta- } \\
\text { das pelo HIV }\end{array}$ & $y$ & $0 \mathrm{~mm}^{-3}$ \\
HIV livres no organismo & $v$ & $10^{-3}$ \\
$\mathrm{~mm}^{-3}$
\end{tabular}

A figura 1 mostra a simulação do modelo utilizando a função (ODE45 do software MatLab $\left.{ }^{\circledR}\right)$. Nessa simulação observamos que modelo representa o comportamento do sistema imunológico na presença do HIV já conhecido na literatura (Perelson and Nelson, 1999; Ho et al., 1995; Chun et al., 1997; Lafeuillade et al., 1996).
Tabela 2: Parâmetros

\begin{tabular}{|c|c|c|}
\hline Parâmetros e Constantes & & Valor \\
\hline $\begin{array}{l}\text { Mortalidade das células } \\
\text { suscetíveis }\end{array}$ & $\mu_{x}$ & $0.02 \mathrm{dia}^{-1}$ \\
\hline $\begin{array}{l}\text { Mortalidade das células } \\
\text { infectadas }\end{array}$ & $\mu_{y}$ & $0.24 \mathrm{dia}^{-1}$ \\
\hline Mortalidade do vírus & $\mu_{v}$ & $2.4 d i a^{-1}$ \\
\hline $\begin{array}{l}\text { Mortalidade das células } \\
\text { defesa }\end{array}$ & $\mu_{z}$ & $0.04 \mathrm{dia}^{-1}$ \\
\hline $\begin{array}{l}\text { Taxa de suprimento das } \\
\text { células suscetíveis }\end{array}$ & $\lambda_{x}$ & $\begin{array}{l}20 \\
d i a^{-1} m m^{-3}\end{array}$ \\
\hline $\begin{array}{l}\text { Taxa de suprimento das } \\
\text { células de defesa; }\end{array}$ & $\lambda_{z}$ & $\begin{array}{l}20 \\
d i a^{-1} m m^{-3}\end{array}$ \\
\hline Taxa de infecção do vírus & $\beta_{v}$ & $\begin{array}{l}2.4 \cdot 10^{-5} \\
m m^{3} \mathrm{dia}^{-1}\end{array}$ \\
\hline $\begin{array}{l}\text { Taxa de ativação da res- } \\
\text { posta imunológica }\end{array}$ & $\beta_{z}$ & $\begin{array}{l}5 \cdot 10^{-6} \\
m m^{3} d i a^{-1}\end{array}$ \\
\hline $\begin{array}{l}\text { Número médio de vírus } \\
\text { livre liberado por uma } \\
\text { célula infectada }\end{array}$ & $k_{v}$ & 360 \\
\hline $\begin{array}{l}\text { Taxa de destruição de cé- } \\
\text { lulas infectadas }\end{array}$ & $p_{y}$ & $\begin{array}{l}0.2 \\
m m^{3} d i a^{-1}\end{array}$ \\
\hline $\begin{array}{l}\text { Taxa de destruição dos } \\
\text { vírus }\end{array}$ & $p_{v}$ & $\begin{array}{l}0.2 \\
m m^{3} \mathrm{dia}^{-1}\end{array}$ \\
\hline
\end{tabular}

\section{Pontos de Equilíbrio}

Os pontos de equilíbrio do sistema dinâmico (1) são dados pela relação:

$$
\begin{aligned}
P & =\left(\bar{x}, \bar{y}, \bar{v}, \bar{z}, \bar{z}_{a}\right) \\
& =\left(\frac{\lambda_{x}}{\mu_{x}+\beta_{v} \bar{v}}, \bar{y}, \bar{v}, \frac{\lambda_{z}}{\mu_{z}+\beta_{v} \bar{v}}, \frac{\lambda_{z} \beta_{z} \bar{v}}{\mu_{z}\left(\mu_{z}+\beta_{v} \bar{v}\right)}\right)
\end{aligned}
$$

onde:

$$
\bar{y}=\frac{\lambda_{x} \beta_{v} \mu_{z}\left(\mu_{z}+\beta_{v} \bar{v}\right)}{\left(\mu_{x}+\beta_{v} \bar{v}\right)\left[\mu_{y} \mu_{z}\left(\mu_{z}+\beta_{v} \bar{v}\right)+p_{y} \lambda_{z} \beta_{z} \bar{v}\right]} \cdot \bar{v}
$$

$\mathrm{ou}$

$$
\bar{y}=\frac{\left[\mu_{v} \mu_{z}\left(\mu_{z}+\beta_{v} \bar{v}\right)+p_{v} \lambda_{z} \beta_{z} \bar{v}\right]}{k_{v} \mu_{z}\left(\mu_{z}+\beta_{v} \bar{v}\right)} \cdot \bar{v} .
$$

\subsection{Ponto de Equilíbrio Trivial}

Para uma pessoa não-infectada pelo HIV temos $\bar{v}=0$. Substituindo isso em (2), então o ponto de equilíbrio trivial é dado por:

$$
P_{o}=\left(\bar{x}, \bar{y}, \bar{v}, \bar{z}, \bar{z}_{a}\right)=\left(\frac{\lambda_{x}}{\mu_{x}}, 0,0, \frac{\lambda_{z}}{\mu_{z}}, 0\right) .
$$

\subsection{Ponto de Equilíbrio Não-Trivial}

Para uma pessoa infectada pelo HIV, temos $\bar{v} \neq$ 0 . Com isso, igualando as equações (3) e (4) e simplificando essa equação por $\bar{v}$, encontramos a equação polinomial de grau 3 dada por:

$$
a_{3} \bar{v}^{3}+a_{2} \bar{v}^{2}+a_{1} \bar{v}+a_{0}=0,
$$

onde os coeficientes são dados por:

$$
\begin{aligned}
a_{3}= & \mu_{y} \mu_{v} \mu_{z}^{2} \beta_{v} \beta_{z}^{2}+\mu_{v} \mu_{z} \beta_{v} \beta_{z}^{2} p_{y} \lambda_{z} \\
& +\mu_{y} \mu_{z} \beta_{v} \beta_{z}^{2} p_{v} \lambda_{z}+\beta_{v} \beta_{z}^{2} p_{y} p_{v} \lambda_{z}^{2},
\end{aligned}
$$



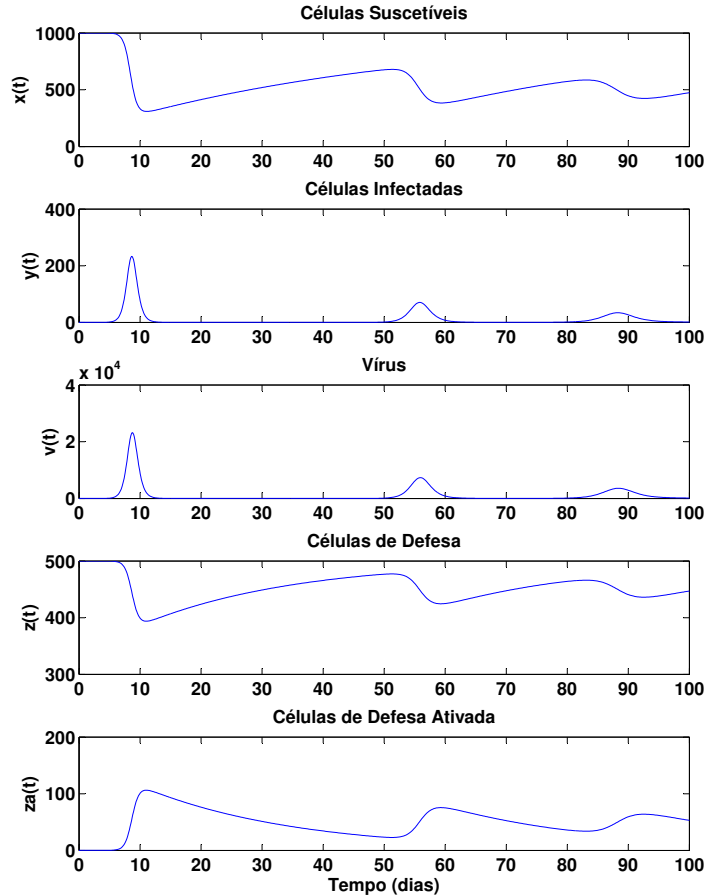

Figura 1: Simulação Numérica

$$
\begin{aligned}
a_{2}= & 2 \mu_{y} \mu_{v} \mu_{z}^{3} \beta_{v} \beta_{z}+\mu_{v} \mu_{z}^{2} \beta_{v} \beta_{z} p_{y} \lambda_{z} \\
& +\mu_{y} \mu_{z}^{2} \beta_{v} \beta_{z} p_{v} \lambda_{z}+\mu_{x} \mu_{y} \mu_{v} \mu_{z}^{2} \beta_{z}^{2}+ \\
& +\mu_{x} \mu_{v} \mu_{z} \beta_{z}^{2} p_{y} \lambda_{z}+\mu_{x} \mu_{y} \mu_{z} \beta_{z}^{2} p_{v} \lambda_{z} \\
& +\mu_{x} \beta_{z}^{2} p_{y} p_{v} \lambda_{z}^{2}-\mu_{z}^{2} \beta_{v} \beta_{z}^{2} k_{v} \lambda_{x}
\end{aligned}
$$$$
\begin{aligned}
a_{1}= & 2 \mu_{x} \mu_{y} \mu_{v} \mu_{z}^{3} \beta_{z}+\mu_{x} \mu_{v} \mu_{z}^{2} \beta_{z} p_{y} \lambda_{z} \\
& +\mu_{x} \mu_{y} \mu_{z}^{2} \beta_{z} p_{v} \lambda_{z}+\mu_{y} \mu_{v} \mu_{z}^{4} \beta_{v} \\
& -2 \mu_{z}^{3} \beta_{v} \beta_{z} k_{v} \lambda_{x}
\end{aligned}
$$$$
a_{0}=\mu_{x} \mu_{y} \mu_{v} \mu_{z}^{4}-\mu_{z}^{4} \beta_{v} k_{v} \lambda_{x} .
$$

A solução da equação (6) foi encontrada com o uso do software MAPLE, porém deixamos a análise dessas soluções para trabalhos futuros.

\subsection{Estabilidade}

Pelo teorema de Hartman-Grobman (Kreyszig, 1978), podemos dizer que um ponto de equilíbrio é estável, se o sinal da parte real dos autovalores da matriz jacobiana aplicada no ponto, a ser estudado, é negativa. A matriz jacobiana do sistema $(1), J(P)$, é dada por:

$$
\left(\begin{array}{ccccc}
-\mu_{x}-\beta_{v} \bar{v} & 0 & -\beta_{v} \bar{x} & 0 & 0 \\
\beta_{v} \bar{v} & -\mu_{y}-p_{y} \bar{z}_{a} & \beta_{v} \bar{x} & 0 & -p_{y} \bar{y} \\
0 & k_{v} & -\mu_{v}-p_{v} \bar{z}_{a} & 0 & -p_{v} \bar{v} \\
0 & 0 & -\beta_{z} \bar{z} & -\mu_{z}-\beta_{z} \bar{v} & 0 \\
0 & 0 & \beta_{z} \bar{z} & \beta_{z} \bar{v} & -\mu_{z}
\end{array}\right),
$$

calculada nos valores correspondentes aos pontos de equilíbrio.

A matriz jacobiana calculada no ponto de equilíbrio trivial (5) é dada por:

$$
J\left(P_{O}\right)=\left(\begin{array}{ccccc}
-\mu_{x} & 0 & -\frac{\beta_{v} \lambda_{x}}{\mu_{x}} & 0 & 0 \\
0 & -\mu_{y} & \frac{\beta_{v} \lambda_{x}}{\mu_{x}} & 0 & 0 \\
0 & k_{v} & -\mu_{v} & 0 & 0 \\
0 & 0 & -\frac{\beta_{z} \lambda_{z}}{\mu_{z}} & -\mu_{z} & 0 \\
0 & 0 & \frac{\beta_{z} \lambda_{z}}{\mu_{z}} & 0 & -\mu_{z}
\end{array}\right) .
$$

Os autovalores da matriz $J\left(P_{o}\right)$ são as raízes $r$ do polinônio característico $p(r)$ dado por:

$p(r)=-\left(\mu_{x}+r\right)\left(\mu_{z}+r\right)^{2} \cdot\left|\begin{array}{cc}-\mu_{y}-r & \frac{\beta_{v} \lambda_{x}}{\mu_{x}} \\ k_{v} & -\mu_{v}-r\end{array}\right|$.

Observe que $-\mu_{x} \mathrm{e}-\mu_{z}$ são autovalores negativos da matriz jacobiana $J\left(P_{o}\right)$. Os outros autovalores são obtidos através da solução da equação do segundo grau dada por:

$$
\left(\mu_{y}+r\right)\left(\mu_{v}+r\right)-\frac{k_{v} \beta_{v} \lambda_{x}}{\mu_{x}}=0
$$

isto é,

$$
r^{2}+\left(\mu_{y}+\mu_{v}\right) r+\left(\mu_{y} \mu_{v}-\frac{k_{v} \beta_{v} \lambda_{x}}{\mu_{x}}\right)=0 .
$$

As soluções de (11) são dadas por:

$$
\begin{aligned}
& r_{1}=\frac{-\left(\mu_{y}+\mu_{v}\right)-\sqrt{\Delta}}{2} \mathrm{e} \\
& r_{2}=\frac{-\left(\mu_{y}+\mu_{v}\right)+\sqrt{\Delta}}{2},
\end{aligned}
$$

onde

$$
\Delta=\left(\mu_{y}+\mu_{v}\right)^{2}-4\left(\mu_{y} \mu_{v}-\frac{k_{v} \beta_{v} \lambda_{x}}{\mu_{x}}\right) .
$$

Para que $P_{o}$ seja sempre localmente e assintoticamente estável, iremos analisar $\Delta$ :

Se $\Delta<0$, então a parte real de $r_{1}$ e $r_{2}$, dada por $\frac{-\left(\mu_{y}+\mu_{v}\right)}{2}$, é negativa.

Se $\Delta=0$, então a $r_{1}=r_{2}=\frac{-\left(\mu_{y}+\mu_{v}\right)}{2}<0$.

Se $\Delta>0$, então temos duas raízes reais distintas, onde $r_{1}<0$. Para que $r_{2}<0$, basta exigir que o produto $r_{1} r_{2}$ seja positivo. Isto implica que:

$$
4\left(\mu_{y} \mu_{v}-\frac{k_{v} \beta_{v} \lambda_{x}}{\mu_{x}}\right)>0 .
$$

Dessa forma, precisamos que:

$$
\frac{k_{v} \beta_{v} \lambda_{x}}{\mu_{x}}<\mu_{y} \mu_{v} .
$$


Com base em (14), definimos o $R_{o}$, a taxa básica de Reprodução, do nosso problema por:

$$
R_{o}=\frac{k_{v} \beta_{v} \lambda_{x}}{\mu_{x} \mu_{y} \mu_{v}}
$$

Se $R_{0}<1$, pelo que acabamos de ver, o ponto de equilíbrio trivial é estável, ou seja, nesse caso, a infecção não se propagaria no organismo do indivíduo.

\section{Conclusões}

Nas simulações numéricas escolhemos o período de 100 dias para observar a fase inicial da doença. O objetivo dessa escolha foi comparar os resultados desse modelo com os já existentes na literatura. Observamos que logo após o período de uma semana, existe um grande aumento do número de vírus e das células infectadas. Além disso, é possível perceber o decaimento das células suscetíveis e das células de defesa efetoras da resposta imunológica. O modelo proposto descreve de maneira aceitável o comportamento do HIV no organismo humano, assim como fazem os demais modelos. O diferencial nesse modelo é podermos analisar separadamente as células de defesa ativadas. Percebemos uma migração das células de defesa para o compartimento das células de defesa ativadas. Acreditamos que isso possa levar, com o passar dos anos, a saturação do sistema imunológico. Além disso, os resultados parecem sugerir uma tendência para um dos pontos de equilíbrio do sistema dinâmico. Dessa forma, em trabalhos futuros, iremos nos aprofundar no estudo dessas questões, com o intuito de sugerirmos um tratamento com o uso de inibidores (medicamentos) por meio do uso da teoria de controle ótimo.

\section{Agradecimentos}

Agradecemos ao CEFET/RJ e o CNPQ por todo apoio financeiro que possibilitou a realização desse trabalho.

\section{Referências}

Amendoeira, M. (2009). Conceitos e métodos para a formação de profissionais em laboratórios de saúde, EPSJV, Rio de Janeiro.

Chun, T. W., Carruth, L., Finzi, D., Shen, X., Digiuseppe, J. A., Taylor, H., Hermankova, M., Chadwick, K., Margolick, J., Kuo, Y. H., Brookmeyer, R., Zeiger, M. A., BarditchCrovo, P. and Siliciano, R. F. (1997). Quantification of latente tissue reservoirs and total body viral load in HIV-1 infection, Nature 387: 183-188. DOI: 10.1038/387183a0
Grégio, J. M., Caetano, M. A. and Yoneyama, T. (2009). State estimation and optimal long period clinical treatment of hiv seropositive patients, Anais da Academia Brasileira de Ciências 81: $3 \quad$ - $\quad 12 . \quad$ DOI: 10.1590/S0001-37652009000100002

Ho, P. P., Neumann, A. U., s. Perelson, A., Chew, W., Leonardo, J. M. and Markowitz, M. (1995). Rapid turnover of plasma virions and CD4 lymphocytes in HIV-1 infection, Nature 373: $123-126$. DOI: $10.1038 / 373123 \mathrm{a} 0$

Komarova, N. and Nowak, M. A. (2001). The evolutionary dynamics of the lexical matrix, Bulletin of Mathematical Biology 63: 451-484. DOI: $10.1006 /$ bulm.2000.0222

Kreyszig, E. (1978). Introductory Functional Analysis with Applications, Wiley.

Lafeuillade, A., Poggi, C., Profizi, N. and et al. (1996). Human immunodeficiency virus type 1 in lymph nodes compared with plasma, $J$. Infect. Dis. 174: 404-407. DOI: 10.1093/infdis/174.2.404

McLean, A. (2013). Infectious disease modeling, in P. Kanki and D. J. Grimes (eds), nfectious Diseases, Springer New York, pp. 99-115.

Nowak, M. and May, R. M. (2000). Virus dynamics mathematical principles of immunology and virology, Oxford.

Perelson, A. S. and Nelson, P. W. (1999). Mathematical analysis of HIV-1 dynamics in vivo, SIAM Rev. 41(1): 3-44 (electronic). DOI: $10.1137 /$ S0036144598335107 